University of Nebraska - Lincoln

DigitalCommons@University of Nebraska - Lincoln

August 1959

\title{
Effect of Change in Moment of Inertia on Line Intensities of Parallel Vibration-Rotation Bands of Symmetric Top Molecules
}

Gordon A. Gallup

UNL,ggallup1@unl.edu

J.L. Koenig

University of Nebraska - Lincoln

Follow this and additional works at: https://digitalcommons.unl.edu/physicsgallup

Part of the Physics Commons

Gallup, Gordon A. and Koenig, J.L., "Effect of Change in Moment of Inertia on Line Intensities of Parallel Vibration-Rotation Bands of Symmetric Top Molecules" (1959). Gordon Gallup Publications. 27. https://digitalcommons.unl.edu/physicsgallup/27

This Article is brought to you for free and open access by the Research Papers in Physics and Astronomy at DigitalCommons@University of Nebraska - Lincoln. It has been accepted for inclusion in Gordon Gallup Publications by an authorized administrator of DigitalCommons@University of Nebraska - Lincoln. 
and $\mathrm{C}-\mathrm{Si}-\mathrm{C}$ angle $\left(111^{\circ} 2^{\prime}\right)$ were obtained from observed rotational constants of $\left(\mathrm{CH}_{3}\right)_{2} \mathrm{SiH}_{2}$.

The rotational spectrum of $\left(\mathrm{CH}_{3}\right)\left(\mathrm{CD}_{3}\right) \mathrm{SiH}_{2}$ was found to consist of closely spaced doublets (see Table II). Tunnel effect splittings due to the $\mathrm{CD}_{3}$ group are immeasurably small and the molecule behaves essentially as a one rotor molecule as far as the microwave spectrum is concerned. From the observed splittings, the barrier was calculated to be $1657 \pm 10 \mathrm{cal} / \mathrm{mole}$.

It is interesting to note that the rotational constants and splittings are consistent with coaxial methyl group and $\mathrm{C}-\mathrm{Si}$ axes (cf. reference 3 ). The agreement between the two $V_{3}$ determinations leads us to believe that accuracy here is limited predominantly by uncertainties in methyl group parameters, and not by neglect of cross terms in the potential energy. We are presently investigating the possibility of setting an upper limit to these cross terms. The results will be published in full at a later date, together with results for other isotopic species, the spectra of which are now being measured.

The author is greatly indebted to Dr. J. D. Swalen and Dr. Dudley R. Herschbach for many helpful discussions.

* This research was supported by a grant from the National Science Foundation.

${ }^{1}$ For a derivation of (1) and definitions see D. R. Herschbach, J. Chem. Phys. 31, 91 (1959).

2 J. D. Swalen and C. C. Costain, J. Chem. Phys. (to be published).

${ }_{3}^{3}$ P. Kasai and R. J. Myers, J. Chem. Phys. 30, 1096 (1959).

1 R. W. Kilb and L. Pierce, J. Chem. Phys. 27, 108 (1957).

Effect of Change in Moment of Inertia on Line Intensities of Parallel VibrationRotation Bands of Symmetric Top Molecules

\author{
G. A. Gallup and J. L. Koenig*
}

Chemistry Department, University of Nebraska, Lincoln, Nebraska

(Received June 8, 1959)

QE EVERAL authors ${ }^{1-3}$ have investigated the degree to which theory can predict the individual line intensities in vibration-rotation bands by considering the first-order corrections due to the moment of inertia change. We wish to report an extension of these calculations to include symmetric top molecules and experimental determination of the change in the moment of inertia effect on the line intensities of the parallel bands of ammonia.

The equations show, as with diatomic and linear molecules, a line intensity dependence on a quantity $\theta_{k}$, which equals $\mu_{0} \xi_{k} / \mu_{1, k}$ where $\mu_{0}$ is the static dipole moment of the molecule, $\mu_{1, k}$ is the rate of change of the dipole moment with the normal vibration $k$ and $\xi_{k}$ is the Taylor's series coefficient in the expansion of the moment of inertia in terms of the normal coordinate $Q_{k}$. The complete intensity formula differs from the zeroorder formula by factors as follows:

$$
\begin{aligned}
& I_{k}^{R} \sim\left[1+\delta_{k}\left(1-4 \theta_{k}\right)(J+1)\right], \\
& I_{k} P \sim\left[1-\delta_{k}(1-4 \theta) J\right],
\end{aligned}
$$

where $\delta_{k}=2 B_{e} / \nu_{k}$.

Measurements were carried out on the parallel bands of ammonia as the total integrated band intensity ${ }^{4}$ and additional molecular parameters are available. ${ }^{5}$ The ratios of the calculated zero-order intensities to the measured intensities for low values of $J$ give experimental values of $\theta_{k}$, from which $\xi_{k}$ can be calculated. The data obtained are as follows:

$$
\begin{array}{cccc}
\text { Mode } & \text { Freq. } & \left.\xi_{k} \text { (calc. }\right) \times 10^{-20} & \left.\xi_{k} \text { (exptl. }\right) \times 10^{-20} \\
\nu_{1} & 3337 \mathrm{~cm}^{-1} & 0.19 & 0.11 / \mathrm{g}^{\frac{1}{2}-\mathrm{cm}} \\
\nu_{2} & 950 & 0.46 & 0.64
\end{array}
$$

These calculations are made on the assumption that other effects are negligible, in particular the Coriolis corrections. Calculations indicate that the Coriolis effect contributes to the intensities only in the second order. These will be reported in a forthcoming paper.

It has been found that sum rules for the $\xi$ 's exist of the form

$$
\sum_{k} \xi_{k}^{2}=1 / I_{B}
$$

This gives a comparison with an independently determined experimental quantity. The $1 / I_{B}$ from infrared data ${ }^{6}$ gives $0.35 \times 10^{40}$ while from the above data it is $0.42 \times 10^{40}$ and the agreement seems satisfactory.

Calculations of the potential function for ammonia using the additional data furnished by the relationship between the $\xi$ 's and the symmetry force constants are being carried out in this laboratory and the results will be reported in a forthcoming article.

* Dupont Postgraduate Teaching Assistant 1958-1959.

1 R. Herman and R. F. Wallis, J. Chem. Phys. 23, 637 (1955).

${ }^{2}$ G. A. Gallup, J. Chem. Phys. 27, 1338 (1957).

${ }^{3}$ H. H. Nielsen, Phys. Rev. 60, 794 (1941).

${ }^{4}$ D. C. McKean and P. N. Schatz, J. Chem. Phys. 24, 316 (1956).

${ }^{5} \mathrm{G}$. Herzberg, Infrared and Raman Spectra of Polyatomic Molecules (D. Van Nostrand Company, Inc., Princeton, New Jersey, 1945), p. 177.

${ }^{6}$ Sheng, Barker, and Dennison, Phys. Rev. 60, 786 (1941).

\section{Comments and Errata}

\section{Note on a Paper by B. F. Gray}

Charles W. Scherr

Department of Physics, The University of Texas, Austin, Texas

(Received January 22, 1959)

CRAY ${ }^{1}$ has recently given an iteration procedure for T for which he has concluded that convergence is limited only by the presence of significant amounts of 
continuum contaminant in the initial wave function. He then points out that no known approximate wave function for the helium atom converges even on the first cycle. The conclusion is that the best available helium wave function ${ }^{2}$ contains appreciable continuum contaminant. That this conclusion is unwarranted ${ }^{3}$ is seen by the following counter-example. Let exp$(1+\delta) r$ be an approximate wave function for the hydrogen atom. Application of Gray's proposed iteration technique leads to a first improved wave function

$$
(1+\delta)^{2} \exp -(1+\delta) r-2 \delta r^{-1} \exp -(1+\delta) r .
$$

The second term is unacceptable for all $\delta \neq 0$. Nevertheless, the weight of the continuum in the initial wave function for small $\delta$ is easily found to be $0.3262{ }_{5} \delta^{2}+$ $0\left(\delta^{3}\right)$; i.e., negligible for $\delta$ small enough. In order to recognize a similar type of error, consider the following only apparently correct argument for another criterion of significant continuum contaminant. Suppose we have an approximate normalized wave function for some system,

$$
\psi=\sum_{n} a_{n} \phi_{n} \text { with } \sum_{n} a_{n}{ }^{2}=1,
$$

where the $\phi_{n}$ are the exact eigenfunctions of the system, and the summation is understood to include an integration over the continuum. With obvious notation, we have the identity

$$
E_{0}^{2}-\left\langle H^{2}\right\rangle=\sum_{n} a_{n}^{2}\left(E_{0}^{2}-E_{n}^{2}\right) .
$$

Now suppose

$$
a_{n} \text { negligibly small for all } E_{n}^{2}>E_{0}{ }^{2} \text {, }
$$

[i.e., Eq. (2) is to be valid for the greater part of the continuum], so that the right side of Eq. (1) is a positive quantity. Thus it follows that

$$
\left\langle H^{2}\right\rangle \leqslant E_{0}{ }^{2} .
$$

From the variational theorem we have (assuming $E_{01}<$ 0 , and $\psi$ reasonably good)

$$
\langle H\rangle^{2} \leqslant E_{0}^{2},
$$

and since the width is necessarily a positive quantity

so that

$$
\left\langle H^{2}\right\rangle \geqslant\langle H\rangle^{2},
$$

$$
\langle H\rangle^{2} \leqslant\left\langle H^{2}\right\rangle \leqslant E_{0}{ }^{2} .
$$

Equation (3) says that a power of the Hamiltonian gives a better energy than the Hamiltonian itself. Equation (3) should now be a test for the presence of significant continuum contaminant. The necessary quantities using the approximate hydrogen wave function above are easily calculated, and it follows rigorously that for no choice of $\delta$ can Eq. (3) be satisfied (except in the physically unrealistic case $\delta<-$ 0.31 ). Nor does Kinoshita's ${ }^{2}$ best wave function satisfy Eq. (3). The source of the trouble in both this argument and in Gray's can only be the assumption of Eq. (2). In both cases the $a_{n}$ have been fixed by the choice of the initial wave function considered, and then, in effect, integrations over a broad spectrum involving the product of these fixed numbers and a quantity which increases without limit (the $E_{n}$ ) are neglected. Thus a wave function whose continuum expansion coefficients do not fall off faster than $E_{n}{ }^{-\frac{3}{2}}$ will fail on Gray's first iteration, and a fortiori on the test of Eq. (3). But this refers to the functional behavior of the expansion coefficients and not to their magnitudes. Indeed, the counter-example shows their magnitudes may be as small as we please; the limiting behavior of its $a_{n}$ for large positive $E_{n}$ and $\delta$ small is of the form $\delta E_{n}{ }^{-\frac{3}{2}}$. This note does not affect the arguments of the first part of Gray's paper which retains its high interest. ${ }^{4}$

\section{B. F. Gray, J. Chem. Phys. 29, 272 (1958).}

2 T. Kinoshita, Phys. Rev. 105, 1490 (1957).

3 The conclusion should now read that this wave function contains some continuum contaminant.

${ }^{4}$ Gray's iteration procedure [as well as my own $\mathrm{Eq}$. (3)] may still be useful of course; e.g., in cases similar to the examples treated by Gray, or to detect, with some ambiguity, the presence of continuum contaminant.

\section{Comment on "Infrared Spectra of Partially Deuterated Diboranes"}

\author{
Walter J. Lehmann and Jerome F. Ditter \\ Research Laboratory, Olin Mathieson Chemical Corporation, \\ Pasadena, Califormia
}

(Received May 6, 1959)

$\mathbf{I}^{\mathrm{N}}$ $\mathrm{N}$ view of recent work on the infrared spectra of monomethyl- ${ }^{1}$ and monoethyldiboranes ${ }^{2}$ we have come to the conclusion that the weak band at 2128 $\mathrm{cm}^{-1}$, rather than $1900 \mathrm{~cm}^{-1}$, in the infrared spectrum ${ }^{3}$ of $\mathrm{B}_{2} \mathrm{H}_{5} \mathrm{D}$ represents the symmetric in-phase $\mathrm{B}-\mathrm{H}^{\prime}$ bridge breathing frequency in the terminally deuterated compound (II). A similar band is observed at 2110 to $2114 \mathrm{~cm}^{-1}$ in the monoalkyldiboranes.

This reassignment would lower the observed TellerRedlich product of I/II for $A^{\prime}$ from 1.84 to 1.64 (see Table VII in reference 3 ). To bring this value back up to $1.84, \nu_{9}$ in compound II is changed from an estimated $940 \mathrm{~cm}^{-1}$ to the observed very weak band at $858 \mathrm{~cm}^{-1}$, while $\nu_{12}$ is reassigned from $858 \mathrm{~cm}^{-1}$ to an estimated $840 \mathrm{~cm}^{-1}$ (unobserved). The net effect of all these changes is an increase in the sum for II+XX (see Table VIII) from 59.4 to 60.1 , in perfect agreement with the sum for I+XXI.

This modification of the assignment results in greater over-all consistency (see Table VI). For instance, a weak band, similar to the $858 \mathrm{~cm}^{-1}$ band in $\mathrm{B}_{2} \mathrm{H}_{5} \mathrm{D}$, is found at $800 \mathrm{~cm}^{-1}$ in the spectrum of $\mathrm{B}_{2} \mathrm{D}_{5} \mathrm{H}$ and has been assigned to $\nu_{9}$ of compound XX; $\nu_{12}$ as in II, is unobserved. Further, the new assignment for $\nu_{2}$ is in accord with the idea that terminal substitution will have relatively little effect on bridge frequencies.

The modified sections of Table VI should now read: 